\title{
More than skills: A novel matching proposal for multiplayer video games
}

\author{
Nadja Stroh-Maraun \\ University of Paderborn \\ Daniel Kaimann \\ University of Paderborn \\ Joe Cox \\ Joe Cox, University of Portsmouth
}

\begin{abstract}
Multiplayer video games are high-involvement products with multiplatform and multiplayer characteristics which aim to enhance player retention by optimizing the matching of teams in accordance with their skills and attributes. However, relatively little academic research has been conducted into the ways in which player attributes can be used to optimize the formation of teams in multiplayer video games. Our study addresses this deficiency in the literature by analyzing a dataset from a popular online multiplayer game that includes historic behavioral data of 6.9 million players participating in 862,664 unique game rounds. We analyze the observable factors associated with longer duration of participation in each round, finding that player retention improves in the presence of player-versus-player combat, variety and heterogeneity. We also show that player retention diminishes as a result of the absence of particular role or vehicle use within a given round. Based on the findings of the analysis, we develop a novel approach called nested matching to assign players to teams with an optimal mixture of skills and inherent and complementary attributes.
\end{abstract}

Keywords: Player Behavior; Matching; Matchmaking; Player Retention; Game Design; Multiplayer Games 


\section{Introduction}

The video game industry represents one of the most significant components of the global entertainment market. According to the Entertainment Software Association, consumers in the US spent more than $\$ 23.5$ billion on game content, hardware and accessories in 2015, an increase of $\$ 1.1$ billion since 2014. Contemporary video game titles (e.g. the Call of Duty franchise) also exceed lifetime sales revenues of all-time movie blockbusters such as Star Wars or Lord of the Rings. Additionally, video games are no longer merely consumed by adolescent males. The Entertainment Software Association also claims that the average gamer in the US is currently 38 years of age, and has been playing video games for about 13 years. Adult female gamers are also shown to represent around twice the proportion of gamers made up by boys aged eighteen or younger [1].

Modern video games are high-involvement products with multiplatform and multiplayer characteristics which aim to deliver long-term entertainment and, consequently, long-term happiness to consumers [2]. This directly results in the retention of consumers, which is often seen as more preferable and profitable than acquiring new ones. Jolley et al. argue that retention can be measured by the duration of time a consumer continues to buy from a company [3]. Rust and Zahorik add that retention can be viewed as the propensity for a consumer to stay with a brand over time [4]. To improve player retention beyond the short term, producers attempt to efficiently and effectively match participants in multiplayer video games into teams and thus customize the video game experience around aspects of the player such as preferences, playing style and skill level [5]. Consequently, this study addresses three key research questions related to enjoyment, matching and retention of multiplayer video games:

(i) Which observable, round-level characteristics affect player retention?

(ii) What are the individual habits and skill levels that affect the efficient matching of players?

(iii) What type of matching algorithms are most efficient in optimizing player retention?

We address these research questions through a multi-stage analysis approach. First, we analyze the factors associated with round-level player retention 
multiplayer video gaming using a unique dataset provided by the Wharton Customer Analytics Initiative (WCAI) and a major international video gaming company. The dataset relates to a popular online multiplayer game and consists of individual player and round-level observations relating to 6.9 million players participating in 862,664 unique game rounds. Following the extensive empirical analysis, we find evidence that players who utilize a wider variety of different roles and vehicles, as well as those who achieve a higher kills-per-second ratio, tend to participate in a greater proportion of the overall duration of any given gameplay round. Second, based on these findings, we develop an approach to player matching that balances the relationship between heterogeneity and homogeneity of player skills, demographics and other attributes to maximize the average proportion of played round completed by players. We use this as a basis for the efficient allocation of players to teams and thus for improving player retention measured by the average proportion of round completed by consumers.

\section{Literature and Hypotheses}

Matching players to form teams in multiplayer settings is an emergent topic in the video gaming literature. Using 200,000 randomly selected and individual game rounds, Myślak and Deja show that the possibility of winning a round is increased by $65 \%$ in instances where perfectly matched teams compete against imperfectly matched teams [6]. The literature on matching (sometimes referred to as matchmaking) mainly focuses on the matching of players into homogenous groups according to their skill level. BuchananOliver and Seo argue that player experience and behavior depend on the interaction with game objectives and rules, which are likely to be affected by variations in consumer skills and abilities [7]. To enhance a player's game experience, various studies have therefore suggested that "best fitted" game rounds should contain similarly skilled players [8, 9]. For example, various studies argue that to balance the game and thus create a challenging, nonfrustrating experience, the probability of winning should be equal for all players $[9,10]$. Véron supports this argument, suggesting that different skill levels would lead to monotonous game sessions due to the superiority of one team [11]. Thus, there exists a consensus in the literature that matching systems should match players according to similar skills.

Nevertheless, an ongoing debate exists on how the skill rating should be constructed. In other contexts, the so-called Elo rating is a rating system for 
chess players that predicts the probability of game outcomes based on skill ratings [12]. This system has been considerably developed and enhanced over time such that it is now used to make similar assessments in a wide range of video games. A particular example of this is the TrueSkill matching system; a Bayesian skill rating system based on the Elo rating which is used on the Microsoft Xbox 360 Live online gaming service [13, 14]. A modification of the Elo rating is also used to investigate the matching of players of equivalent gaming skills in the online game Warcraft III [15]. Delalleau et al. further suggest that a matching procedure should rely on a multi-dimensional skill level identification to generate more balanced matches in the game Ghost Recon Online [9]. This particular study relies on the TrueSkill system as well as other player attributes such as accuracy, number of played sessions or the number of kills, deaths and captures within the game.

A different approach is the Bradley-Terry model which compares pairs and predicts the probability of winning for one of the two individuals [16]. Huang et al. extended the Bradley-Terry model to compare paired teams and provide individual skill results [17]. A similar extension of the BradleyTerry model is also used to balance teams in the game Enemy Territory by reallocating players from one team to the other [18].

Another advanced approach to player matching is proposed after analyzing the online game League of Legends where teams ideally consist of players with different roles [6]. The matching system developed by the authors for this game assigns role-specific skill levels for each player and matches together players with similar skill levels in different roles. A similar result is found by Chen et al. who state that players' skills should depend on the base skills of the players, base skills of the role they are playing and the role-specific skills of the players [19].

Alternatively, Francillette et al. have developed a multilevel matching system whereby players are grouped according to their playing style and they are clustered within a group according to their skill level [8].

Following these studies, we propose that optimal matching of video game players with similar skill levels should create a more challenging and enjoyable game experience and thus maximize the likelihood of player retention within a given round. We therefore formulate the following research hypothesis:

$H_{1}$ : Greater homogeneity of player skill, as measured by variation in player starting rank, death/kill ratio and non-combat score, positively affects 
player retention within a given gameplay round.

It should however be noted that a number of the studies cited above show that player matching should not necessarily be undertaken on the basis of skill level alone $[9,6,8]$. Instead, it is important to recognize that player motivations are multifaceted and are affected by a combination of psychological needs, behavioral patterns and personality traits [20]. Using survey and behavioral in-game data from the game Fallout: New Vegas, Chen et al. show that scores and other aggregated features are of limited value in player matching. Instead, they found that in-game behavior such as sequential patterns might help to predict the player's characteristics and that game expertise is a dominant factor affecting the behavior within the game [21]. Nagle et al. model variations in duration of gameplay in first-person shooters according to personality traits, finding that customization and difficulty adaptation around these personality traits have a significant effect on gameplay duration [22]. An effective approach to player matching should take into account a range of further attributes to optimize the construction of teams such as playing styles and specific roles adopted within a team as part of complex in-game strategies [23]. For example, the player experience is strongly influenced by physical proximity to the gameplay server, which affects response time and thus the length of time a player spends waiting to be assigned to a round [11]. Therefore, geographical factors are likely to influence the extent to which players are willing to spend time participating in game rounds where there is high variation in the physical proximity to the gaming server. Further, evidence from the game Soccerbots shows that effective matching should be based on the clustering of observed behaviors such as roles and playing styles [24].

Shores et al. also establish that deviant behavior in online social systems affects the retention of other players [25]. More precisely, so-called 'toxic' players encourage higher drop-outs from rounds among fellow players in the game League of Legends. A similar argument is made in relation to the game MechAssault, where the authors give evidence that players prefer to be matched on the same team along with players that demonstrate the same characteristics as themselves [26]. Riegelsberger et al. therefore asked players to rank other gamers' profiles which contained different attributes and behaviors. They concluded that one player type prefers to be matched to other players who are basically homogenous in terms of skills whereas another player type prefers players with a friendly gaming personality and 
the last player type prefers pugnacious players. Thus, the alignment of a 'preferred' set of behaviors is likely to represent one desirable feature of an effective matching system, where teams are formed on the basis of characteristics such as variations in player age. Finally, Prugsamatz et al. find consumer attitudes to be the most significant predictor of purchase intentions in the market for video games, particularly distinguishing between 'hardcore' and 'casual' gamers [27]. If players make purchasing decisions on the basis of their attitudes towards gaming, it seems reasonable to also suggest that these same attitudes might also be an important determinant of the gameplay experience when matched with and against other gamers with similar or different attitudes. This makes it important to factor player attitudes into the design of an effective matching algorithm.

Following these studies, we identify a range of individual attributes and historical behavioral data to explain player retention, measured in terms of the average proportion of a given round completed by players within a given round of gameplay. We anticipate that players seek an optimal level of variety among their gaming rounds and thus address the following research hypotheses:

$H_{2 a}:$ Greater heterogeneity among the inherent attributes of players, i.e., roles, vehicles, levels and game modes, positively affects player retention within a given gameplay round.

$H_{2 b}$ : Greater homogeneity among the complementary attributes of players, i.e., age, country and gender, associates positively with player retention within a given gameplay round.

\section{Analysis}

\subsection{The Game}

To address each of the research hypotheses outlined in the previous section, we use a dataset provided by the WCAI and a major international video gaming company. The cross-sectional dataset is collected from a multiplayer first-person shooter game available for two home consoles (Sony Playstation 3 and Microsoft Xbox360) and PC. The dataset consists of information on 862,664 unique rounds, where at least two players are pitted against one another in a combat scenario. The game features the combined arms battles 
across single-player, co-operative and multiplayer modes. The primary objective of a round varies between game modes, although players generally aim to kill opponents and avoid being killed themselves. Each round has a map, duration, and player count. Maps have different goals and possibly afford access to different roles, functions, and abilities for players. Maps differ in terms of physical layout rather than degree of difficulty; such that Map 1 is not necessarily harder than Map 2. The maximum number of players in a round depends on the hardware platform on which the game is being played, with an absolute maximum of 64 players in a round at any given point. New players can join the round at any time until the maximum threshold is reached, at which point new players may only join to replace participants who choose to leave the game round before its conclusion. Players who are killed are able to 'respawn' within the game round and continue playing after a short delay. Players also select a 'role' by adopting a particular player class (e.g. engineer or recon) with a particular set of gameplay attributes and abilities and are able to change their role upon respawning.

Players can start a gaming round by either manually choosing a particular server or selecting the 'instant play' option that matches the player to a currently active round. When choosing a server manually, players have complete information about the number of active participants, the specific settings of the round and the performance of any players currently participating. By contrast, players who select the 'instant play' feature have incomplete information about the gaming round. Thus, the matching algorithm used by the game should use available information to match players to rounds and teams according to the server characteristics and round settings, as well as to the performance and preferences of the player. As the game is not a particularly social one, the dataset does not include specific information about player interaction and instances where multiple rounds are played along with the same people are relatively rare. Accordingly, people tend to play independently, so the matching algorithm is predominantly responsible for matching players to teams and/or servers.

The game additionally features a range of different roles and vehicles. Players can choose from four different roles: assault, engineer, support, and recon. The assault role represents the 'middle ground', promoting both offensive and defensive play styles. Accordingly, the primary weapon of the assault role - the assault rifle - is a versatile weapon that can engage the enemy at any range. The engineer role features a repair tool to repair friendly vehicles as well as numerous shoulder-fired missiles and anti-tank mines to 
combat enemy vehicles. The primary weapon of the engineer is the carbine, a shorter version of assault rifles allowing for close to medium range combat. The support role represents the definition of 'team player', with a selection of weapons and gadgets that are primarily focused on supporting the team. The primary weapon of the support class is the light machine gun, which has a high ammo capacity, high power over long ranges, but poor accuracy. The recon role is for stealthy players gathering reconnaissance on enemies and moving is undetected. Recon contains battle rifles, marksman rifles, and sniper rifles. These rifles inflict the most damage of any weapon in a single shot. They are also the most accurate, which is beneficial when firing at enemies at long ranges. As a trade-off, both of these benefits are weighed against the substantially longer time it takes to fire successive shots in comparison to when other weapons are used. In addition to these four distinct player classes, the game features over twenty different vehicles that can be used in each round, split into twelve different classes ranging from ground vehicles (e.g. jeeps, transport, infantry fighting vehicles, main battle tanks), aircraft (e.g. helicopters, jets, gunships), static emplacements (e.g. stationary anti-air systems), and vehicular accessories (e.g. anti-air missiles, light machine guns). The game also features a total of 145 ranks. A set amount of experience must be earned from combat and non-combat actions to level up to a higher rank. After leveling up, players are able to unlock access to new specializations or weapons.

\subsection{Phase One: Players' Historical Behavior}

Our dataset consists of observations for all game rounds in which multiple players participate simultaneously. It includes several round-level characteristics such as the duration of the round, the game mode, the number of participating players and the particular map/level being played. Means and standard deviations of key variables are calculated based on the complete information recorded for the individual players participating in each round. Our dataset contains 30,156,196 observations relating to 862,664 unique gameplay rounds and 6,884,986 players. About $90 \%$ of all rounds end after at most 48 minutes and $99 \%$ of all rounds end after at most 90 minutes. As players are able to leave a gameplay round ('drop out') at any time, we analyze the average proportion of the round completed by participants at the point they 'drop out'. We assume that higher values of the mean proportion of round completed represent higher levels of player retention, since a greater proportion of players choose to participate in a greater 
proportion of the total round duration. In other words, the drop-out rate is lower for rounds that demonstrate a higher average completion. We model variations in the average proportion of a round completed at the level of individual rounds $r$ as a function of player skills, player attributes and round characteristics and specify the following round level model (Equation 1):

$$
\begin{aligned}
\text { ProportionRoundComplete }_{r}= & \alpha+\beta \text { GamingSkills }_{r} \\
& +\gamma \text { InherentAttributes }_{r} \\
& +\delta \text { ComplementaryAttributes }_{r} \\
& +\epsilon \text { Controls }_{r}+\eta
\end{aligned}
$$

where Proportion RoundComplete represents the average percentage of round $r$ completed by the participating players. GamingSkills is a vector of variables controlling for the level and variation in player skill; consisting of the average and standard deviation of the participants' rank at the start of round $r$, the average and standard deviation of the kills per second in round $r$, and the average and standard deviation of the score earned by players in a given round $r$ derived from non-combat actions (e.g. healing or repairing). InherentAttributes is a vector of variables capturing the proportion of different roles players adopted during the round $r$ (i.e. assault, recon, engineer, and support), the proportional use of vehicles in round $r$ (i.e. armored and unarmored land vehicles, jet, boat, and helicopter), the game mode of round $r$ (i.e. Conquest, Rush, and Team Death Match), and the total number of players engaged in round $r$. ComplementaryAttributes is a vector of variables containing measures of the average and standard deviation of the age of players participating in round $r$, the number of individual countries in which participating players are based, and the proportion of male players.

Descriptive statistics relating to each of the variables reflecting the roundlevel data can be found in Table A.1.

[Table A.1 about here]

The data shows that the mean proportion of a given round completed by players is around $58 \%$, meaning that, on average, participation in a particular round is for just over half of its overall duration. It also appears that a greater proportion of players in a typical round adopts the 'engineer' and 'assault' roles than the roles 'recon' and 'support'. Further, it can be observed that a 
relatively high proportion of players tend to make use of vehicles; the most popular being land-based vehicles and helicopters and with a small minority electing to use a jet or water-based vehicles. The standard deviation of vehicle usage between rounds tends to be relatively high due to the impossibility of using particular vehicles in certain combinations of game modes and levels. Our data also shows significant disparity in the popularity of the various game mode options that define player objectives, with the most popular by far being 'Conquest', 'Rush', and 'Team Death Match'. By contrast, it appears there is much greater variety in the particular map/level selected for each round. In terms of the demographic characteristics recorded for all players participating in each round, it appears that the average player of this game is a male in his late 20s, with relatively low levels of variation observed around this age. A typical game round also involves players from around 11 different countries.

\subsection{Results}

Table A.2 contains a set of ordinary least squares (OLS) regression results based on the round-level data described above. In each case, the dependent variable is the average proportion of each round completed by participants. Model Specification (I) contains all of the independent explanatory variables listed in Equation 1 above, while model Specification (II) additionally contains quadratic terms for a majority of variables to test for the possibility of a non-linear relationship with the proportion of round completed.

\section{[Table A.2 about here]}

In relation to the vector of variables controlling for player skill level, the clearest result relates to the strong, positive coefficient estimated for the number of player kills within a given round per second of gameplay. This variable is a proxy for the intensity of player-versus-player combat occurring within a given round and shows a preference for this form of player interaction at the group level. We also see that, on the one hand, higher ranked groups of players tend to participate in a slightly lower proportion of rounds on average in comparison with lower ranked groups, which may indicate that higher ranked players are marginally more selective or opportunistic in terms of their behaviors and preferences. On the other hand, a higher variation in starting rank is found to associate with an linear increase in the proportion of 
a given round that is completed. But although the estimates are statistically significant, the coefficients themselves are so small as to suggest that this relationship is negligible.

The results also indicate that combat-related activities associate with greater proportions of round completion than non-combat related activities. Specifically, we find a significantly positive relationship between player retention and the mean number of kills-per-second recorded by players participating in the round. A one unit increase in the average kills-per-second increases the average proportion of round completed by around $28 \%$. However, the negative coefficient estimated for the quadratic term shows the relationship to be concave; in other words, as the average number of killsper-second increases, the positive effect on player retention diminishes. By contrast, the standard deviation of this variable is shown to have a significant and negative impact on player retention such that if players differ in the rate at which they are able to kill others within the game, their propensity to remain in the round diminishes. This relationship is also shown to be non-linear such that the reduction in player retention becomes greater the more the rate of kills differ between players. The results further indicate a much lower coefficient estimate for the mean non-combat score earned in each round, which indicates that players collectively enjoy having greater opportunities to increase their score via combat in comparison with non-combat actions. Overall, these findings appear to offer support for hypothesis $\mathrm{H}_{1}$ and suggest that skill is an important determinant of player retention. Thus, our study confirms former studies that all show the importance of homogenous skill levels for player retention $[8,9,11]$.

As far as player attributes are concerned, we find evidence to suggest that the maximum player count has a small, positive impact on the average proportion of rounds completed. This may broadly support the contention that players have a preference for action, since a higher active player count is likely to increase the opportunities for scoring via combat. All of the coefficient estimates relating to the use of roles and vehicles suggest that greater proportions of players adopting each role or vehicle during a round result in longer player retention. For example, a one unit increase in the proportion of the used assault role increases the proportion of round completed by $4.3 \%$, whereas a one unit increase in the proportion of the jet vehicle is found to associate with an increase in the porportion of round completed of $5 \%$. However, the results from the non-linear estimation in Specification (II) suggest that groups of players typically experience diminishing returns to higher pro- 
portions of players adopting both each role or vehicle. Thus, the relationship between player retention and the proportions of players using the range of available roles and vehicles tends to follow an inverted ' $u$ '-shaped pattern.

Taken together, this suggests that groups of players tend to value action and variety in preferring to play in rounds where each role and vehicle is adopted by a greater proportion of players at some point during the game round. In other words, the scarcity of particular roles or vehicles from a given gameplay environment tends to associate with reduced levels of player retention. We also show evidence that different game modes and levels affect player retention. Specifically, conquest modes and death matches seem to universally associate with lower average retention in comparison to other modes, most notably rush matches. This further supports the contention that players value combat-related scoring. Overall, these findings offer support for both $\mathrm{H}_{1}$ and $\mathrm{H}_{2 \mathrm{a}}$, as well as findings elsewhere in the literature on player retention in video games $[9,8,6,27]$.

Our results further show that player groups with higher average ages tend to complete greater proportions of rounds in comparison with groups who are younger on average. We also show that the relationship is essentially linear, with a very small but statistically significant coefficient estimate for the associated quadratic term. Additionally, we find that a higher standard deviation of player ages within a game round associates with a marginal reduction in player retention. We also find evidence to suggest that rounds in which a greater proportion of the players are male tend to result in a reduction in player retention, which implies that players typically value gender diversity. Finally, we show an essentially linear, negative relationship between the proportion of a round completed and the count of the number of players participating in that round from different countries. A one unit increase in the number of participation countries in one round decreases the proportion of round completed by around $0.5 \%$. This finding may lend support to arguments made above relating to cultural and language barriers affecting the gameplay experience, as well as a distaste for time-delays or 'lag' caused by greater geographical distances between players [11]. Overall, this evidence offers support for hypothesis $\mathrm{H}_{2 \mathrm{~b}}$ and suggests that the aggregate demographic profile of players participating in a given game round has a significant effect on retention.

The round-level analysis presented in this section suggests that player skills and attributes associate significantly with player retention. Indeed, groups of players seem to enjoy participating in high scoring rounds, with a 
preference for score enhancement from combat rather than non-combat activities. Game rounds also seem to enjoy higher retention levels when greater numbers of players are involved simultaneously, which further suggests that players value more active, combat-related interactions. Our findings related to the use of roles and vehicles also suggest that player groups value variety and suffer disutility from the absence of particular roles or the lack of vehicle use within a given round. In addition, and with respect to characteristics and demographics, we show that player retention is associated with a greater extent by homogeneity in the composition of the group with which they are matched in any given game round.

\section{Phase Two: Nested Matching}

The findings outlined in the previous section show that heterogeneity in player demographics, gaming skills and other attributes (i.e. roles, vehicles, levels, and game modes) associate significantly with variations in retention levels within a given gameplay round. In this section, we use the results from this previous analysis to design a procedure that matches new players to existing rounds in such a way as to increase the proportion of each round completed. The matching procedure we employ, called nested matching, uses a multi-step approach to group new players into existing teams by systematically reducing the number of possible rounds to which they could be efficiently attached. We do this by forming homogenous groups of players not only according to their skill levels but also to their attributes. Therefore, our matching algorithm is unique in comparison with others used elsewhere in the literature that mainly focuses on 'skill level matching' and fail to either use a multi-step approach or to incorporate simultaneously different elements of player skill and other attributes $[9,8,10]$.

We base our assessment of player behavior such as vehicle use and role choice on a weighted average of their activity recorded over the previous $n$ rounds, where $n$ should be sufficiently small. We also assume that players enter the game sequentially. Figure B.1 illustrates the nested matching procedure and outlines the three matching steps, namely round-characteristic matching; skill matching; and attribute matching. The round-characteristic matching step reduces the number of potential rounds by identifying those with spare capacity and grouping by gaming hardware platforms. This essentially represents a pre-step and happens before new players enter the game. The second step of skill matching identifies a further reduced sub-set 
of rounds which the new incoming player could be efficiently matched to. In the final step of attribute matching, the incoming player is matched to a round within the group that maximizes the 'fitting quality' of her skills and attributes with the previously identified round characteristics, thus maximizing the likelihood of retention within the round to which she has been attached. These steps are outlined in greater detail below.

[Figure B.1 about here]

\subsection{Step 1: Round-characteristic matching}

Before a player enters the game, it is necessary to reduce the number of possible rounds to which she could be attached to minimize the matching time. Thus, the first step in our nested matching algorithm involves identifying all rounds with spare capacity and grouping them according to the gaming hardware platform. Spare capacity is defined in this instance as a round in which at least one additional player can join. If a new player wants to join the game, only rounds with spare capacity are eligible for assessment using the matching procedure, with all other rounds omitted. The total maximum number of players allowed to participate in a single round also depends in part upon the hardware platform on which the game is played. As each round can only be played on one specific gaming platform, we divide the rounds in three groups, namely PC, PS3, and XBOX360. It is not possible for players using different hardware platforms to compete against each other within the same game round.

After the first matching step has been completed, there exist three groups of rounds, one for each platform, to which incoming players can be matched, which we refer to as 'platform groups'. In subsequent matching steps, we only consider one of these groups dependent upon the hardware platform being used by the individual player.

\subsection{Step 2: Skill Matching}

According to the analysis of round-level data presented in Section 3, the variables that show the strongest relationship with player retention are the mean and standard deviation of the number of kills-per-second recorded by players participating in that game round. These imply a positive but diminishing impact on player retention associated with a higher average number of 
kills-per-second. We also find evidence that a higher standard deviation for this variable associates increasingly negatively with player retention. Thus, it is important to group players into rounds with the goal of achieving the greatest degree of homogeneity according to the number of kills-per-second they achieved in previous rounds. Our analysis has also shown that other factors such as non-combat related scoring and the player's overall rank relate only negligibly to player retention in the analysis presented in Section 3. When a new player wants to join a round, her skills measured in terms of the average number of kills-per-second recorded in previous rounds in comparison to all mean skills within the rounds in the platform group. If the average number of kills-per-second being observed in a particular round is similar to the player's average number of kills-per-second in the previous rounds, the current round is considered to be a feasible match for the incoming player. Consequently, to speed up the matching procedure of each new player, existing rounds are sorted according to their mean kills-per-second into skill groups which are subgroups of the platform group. Each group therefore contains a more limited range of mean kills-per-second.

The matching of a new player to rounds on the basis of her skill level is in line with the existing literature on matching in multiplayer games $[10,8,9]$. However, our approach differs significantly from other studies given our use of skill level matching only as a filtering mechanism by which to find the broad 'skill group' or set of rounds where the skill level of the opponents is similar to the player's own. To select the specific round within this skill group to match the player to, we employ a final stage of matching according to individual attributes.

\subsection{Step 3: Attribute matching}

In the final step of our matching procedure, we match individual players to a specific gameplay round from within the feasible set identified from the previous steps. As we have showed in the analysis presented in Section 3 , retention levels not only differ according to skill and use of hardware platform, but also in terms of their inherent complementary attributes. Most existing matching approaches for multiplayer video games do not account for these factors. Thus, the third and final step of our nested approach involves matching players according to their identified attributes. While the statistical significance and magnitude of estimated coefficients for these variables were previously shown to be smaller than those for variables representing player skill, the aggregate effect of attributes in terms of variation in player retention 
is non-negligible and thus should be accounted for as part of the matching process.

Our earlier analysis has shown that players favor playing different levels and game modes, as well as using roles and vehicles. More precisely, the proportions of players adopting different roles indicate an inverted u-shaped relationship with player retention. This means that it is possible that an incoming player adopting a particular role might lead to a reduction in retention within that round if a relatively high number of other players are already adopting the same role. On the other hand, the addition of the same player and role may lead to an increase in retention if a relatively small number of other players are adopting the same role.

Players also differ in their complementary attributes or demographics, especially with respect to their age and country of residence. As our analysis has shown, retention is adversely affected by greater variations in both the ages and countries of residence of players participating in the same gameplay round. Although coefficient estimates for both variables are relatively small in magnitude, they are also highly statistically significant and show nonnegligible effects on player retention if the variations in these variables are particularly high. Thus, our procedure attempts to match players with others of a similar age and minimize the number of players from different countries participating in the same round. In summary, we match players to teams based upon their attributes, which are measured according to their recent choices of levels, game modes, roles, and vehicles as well as their age and country of residence.

Bringing the three steps together, our nested matching algorithm suggests that if a new player $i$ wants to join a round $r$, she should be matched to those which offer the most suitable characteristics within the broad skill group outlined in Step 2. As we match according to a vector of attributes, it might not be possible to identify a perfect match in accordance with every variable. However, our algorithm makes it possible to compensate any 'misfitting' of one variable by achieving a close match with another. Specifically, we assume additivity between the fits of the variables. By weighting these fits, we take into account that some attributes are associated with a higher increase in the proportion of a round completed (see Section 3). For example, as the empirical analysis has shown that game modes have a larger coefficient than age, game modes should also be given a higher weight than age in the attribute matching. Thus, heterogeneity in age is easier to compensate than the misfitting of a game mode by using a weighted sum that gives a smaller 
weight to age than to game modes. Therefore, we model the overall fitting quality of a player $i$ into a round $r$ as a weighted sum of the attributes we have found to be significant for player retention:

$$
\begin{aligned}
\text { Fit }_{i r}= & a_{1} \text { Age }_{i r}+a_{2} \text { Country }_{i r}+a_{3} \text { Level }_{i r} \\
& +a_{4} \text { Mode }_{i r}+a_{5} \text { Role }_{i r}+a_{6} \text { Vehicle }_{i r}
\end{aligned}
$$

where the attributes represent dummies. They ask whether the range of ages and numbers of countries represents an appropriate fit $\left(A g e_{i r}\right.$ and Country respectively); whether the preferred level is used $\left(\right.$ Level $\left._{i r}\right)$; whether the preferred game mode is played $\left(M_{o d e}\right)$ and whether the player's preferred role and vehicle use is still required (Role $e_{i r}$ and Vehicle $_{i r}$ respectively) in round $r$ if player $i$ is matched to it. Thus, by weighting the variables and summing them up $\left(a_{1}, \ldots, a_{6} \in(0,1), \sum_{j=1}^{6} a_{j}=1\right)$, we derive a value between 0 and 1. We interpret the fitting quality $F i t_{i r}$ as a percentile measure. The higher the fitting quality, the better the round matches the player's attributes. We are looking for the round $r^{*}$ which fits the player's attributes best. Thus, the player $i$ is matched to the round $r^{*}$ where the fitting quality is maximized

$$
r^{*}=\underset{r}{\operatorname{argmax}} F i t_{i r} .
$$

The existing literature on matching in multiplayer games neglects the final 'attribute matching' step of our approach almost completely. Limited attempts to, at least partly, account for player attributes in matching procedures can be found in $[24,26]$, although our nested matching approach is the first to fully incorporate a combination of both player attributes and demographics within a single algorithm. However, it should be noted that one limitation of our method is the assumption that players enter the game sequentially, which makes matching individual players to the best-fitting round a more straightforward process. To find a 'good' solution in instances where this is not the case, we suggest the combination of methods from classical economic matching theory such as the deferred acceptance algorithm which yields an optimal and stable solution for new players [28] with our approach of nested matching. In this case, instead of simply choosing a player's round by maximizing the fitting quality, we use this measure to form preference lists and then apply a matching algorithm to match players into a round.

To test the validity of the nested matching in comparison to a simpli- 
fied skills-based matching approach, it would be ideal to actually implement the model and evaluate its performance. However, the current collaboration with the international video gaming company does not allow such an implementation. Nevertheless, we perform a predictive validation to test our model and evaluate its explanatory power. Shmueli and Koppius state that predictive validity analytics offer a straightforward way to compare by examining their predictive accuracy [29]. The study on comparison of diffusion models with simpler linear models for forecasting information system spending by Collopy et al. provides a good example of this usage [30]. Another example is the study by Sethi and King, which used predictive analytics to compare linear and nonlinear models for obtaining user information satisfaction [31]. Finally, Chen et al. use predictive validation to develop frequent pattern mining algorithms and show that a properly selected predictive model with regularization and cross validation can help confirm the validity of results [21]. We undertake comparative predictive validation of the skills-based matching and our nested matching by performing $\mathrm{k}$-fold cross validation tests. More precisely, we compare the matching procedure actually used in the game with our new approach. The actual matchmaking is based on a score which mainly captures the combat-related actions but also reflects some non-combat achievements. The sample was randomized and split into two subsamples, a training and a validation sample. The results of the validation exercise are shown in Table A.3. Both the skills-based and nested matching show a reasonably good predictive validity, with an increase of approximately 3.3 percentage points in average predictive validity of the nested matching relative to the skills-based matching. In terms of the fit validity, the nested matching shows an acceptable $\mathrm{R}^{2}$ of approximately $21 \%$ whereas the skills-based matching only presents a $7 \%$ fit quality. Altogether, we can summarize that the nested matching outperforms the skills-based matching and thus better captures the habits and preferences of players. Thus, the nested matching approach is closer to reality and equally adds research and managerial value to both academics and practitioners.

[Table A.3 about here]

\section{Discussion}

Our analysis has shown that both skills and attributes matter and thus should be considered when matching players. Ours is the first study that 
considers the nested matching technique involving a three-step approach to match players into teams. While our findings are based on data from a single first-person shooter game, it is likely that this kind of matching would also be useful in other games. We draw on evidence from the literature which suggests that matching approaches based on player skill levels alone are not appropriate and that the quality of a matching can be improved by considering additional player attributes.

Delalleau et al. use an experimental setting using an early Ghost Recon Online beta test to compare levels of fun (expressed by the players in a survey) when different matching algorithms are used [9]. They test two variants of the TrueSkill rating models against machine-learning techniques that use the players' fun levels as a means to match teams. They show that fun-based matching outperforms the TrueSkill rating models. More specifically, while the TrueSkill matches were ranked according to the probability that team A wins, the fun matches were ranked according to the probability that the players express that they are having fun in the game. It is shown that Kendall's tau of the fun matches was higher (0.23) than that of the others (0.11 and 0.17 respectively). Although the study does not provide a precise definition of 'fun', it does offer clear evidence that players have different preferences and should be matched according to factors beyond skill levels such as other 'softer' factors or preferences that we use in our nested matching approach.

There is also evidence from Multiplayer Online Battle Arena (MOBA) games, that nested matching is a promising approach which is worth considering in many different types of game. MOBAs belong to the game genre eSports where two teams engage in combat with the help of a chosen avatar, often called a 'champion'. Popular MOBA games include League of Legends (LoL) and Dota2. Each of the available champions has special skills and weaknesses and thus a MOBA game is characterized by offering strategic decision making in addition to skill-based combat [19]. Myślak and Deja provide evidence that a matchmaking approach which considers the players' preferences over roles or champions would improve the chances of winning for the team in LoL [6]. More precisely, the authors counted the number of players in each team who played on their favorite position and estimated a logit model with the dependent variable reflecting whether or not the team won or lost. They show that a team's chances of winning increase with each additional player playing on his or her favorite position within the team and decreases when such a player is on the opposing team. When a team that is perfectly matched regarding the roles played against a team where no player 
adopts their favorite role, the probability of winning is greater than $65 \%$ for the perfectly matched team. A nested matching approach such as the one advanced by this study can incorporate factors such as the adoption of a favored role when allocating players to particular teams.

Jiménez-Diaz et. al. find evidence that skill-based matchmaking in multiplayer online games should be developed and extended "with information about the particular abilities of the players that make them more apt for playing a given role in the team". By employing clustering methods in the context of the game Soccerbots, where two teams of robots compete in a soccer game [24], the authors argue that players should be matched according to the extent to which players adopt primarily defensive behaviors. Our nested matching approach could be used in this setting to match players first according to skills and then in terms of the different roles (e.g. goalkeeper, defender or midfield player) that are necessary to form a defensive team. Accordingly, this correlates with our findings that players value a certain level of heterogeneity regarding the different roles a player can adopt.

Thus, game designers should be aware of the fact that a matching algorithm that only considers player skill might not be appropriate for all games. Instead of developing more and more complex skill-based ratings, it might be advisable to investigate a wider range of factors and attributes that might influence the efficient matching of players to teams. By using a variant of the nested matching which incorporates the wider characteristics of players in the context of the specific gameplay environment they are able to improve the quality of matches within the game and consequently enhance player retention.

\section{Conclusion}

A key contribution of this research is the design of a novel 'nested matching' approach with the goal of maximizing player retention at the level of the gameplay round. Through the use of a detailed and large-scale dataset, our findings significantly extend the literature on consumer engagement with multiplayer video game experiences [32] and on matching in multiplayer games $[33,26,10]$, which until now has only partially examined the preferences and attributes of players when considering optimal approaches to matching and team composition. When developing our nested matching procedure, we identify a number of player and gameplay traits (i.e. demographics, characteristics, skill levels, and attributes) consistent with prior 
studies [34] and find both player attributes and skill levels to be significant predictors of player retention. Our model of player retention demonstrates that players not only value combat-related actions, but also prefer variety in their use of gameplay elements such as roles and vehicles. However, the non-linear relationship between these variables and our measure of player retention show that an optimal mix between homogeneity and heterogeneity of player and gaming characteristics should be combined to enhance player retention within a given round of gameplay.

Regarding limitations, this study stills lacks a real-life evaluation of the nested matching algorithm which is based on the empirical results and incorporates information that other algorithms do not. To test the validity of nested matching, we perform predictive validation tests. However, a real-life testing of the algorithm would be helpful to determine whether this instantiation makes a difference for game designers and game studio distributors. It may be that the randomness of the matching is a problem on its own and that even with the new identified variables there is no significant change in duration of play or quitting and thus player retention.

As this work is the first that considers the nested matching technique involving a three-step approach to match players into teams, the study of the applicability of the nested matching approach for other games is needed. Although skills and attributes are important factors of matching in other games (e.g. MOBA games), additional research is necessary to identify the exact set of variables that should be considered in a matching. Additionally, the considered game is not a particular social one. We found that players should be homogenous concerning age and countries. If social aspects are more important, it might be beneficial to match players that differ in age and home origin to consider cultural aspects.

Future studies could build on this work by studying the role of other players' behavioral preferences and interactions (e.g. the achievement of objectives) to achieve better and more advanced matching procedures. It would also be valuable for future studies to investigate the impact of different matching procedures on the gameplay experience more directly (as opposed to the indirect measure of experience/retention used here), as well as the extent to which team performance affects player interaction and retention. 


\section{References}

[1] Entertainment Software Association, Essential facts about the computer and video game industry, available at http://www.theesa.com/aboutesa/essential-facts-computer-video-game-industry/Essential-Facts2016.pdf Accessed: 2nd May 2017 (2016).

[2] E. W. Anderson, C. Fornell, D. R. Lehmann, Customer satisfaction, market share, and profitability: Findings from sweden, Journal of Marketing 58 (3) (1994) pp. 53-66.

[3] B. Jolley, R. Mizerski, D. Olaru, How habit and satisfaction affects player retention for online gambling, Journal of Business Research 59 (6) (2006) $770-777$.

[4] R. T. Rust, A. J. Zahorik, Customer satisfaction, customer retention, and market share, Journal of Retailing 69 (2) (1993) 193 - 215.

[5] S. Karpinskyj, F. Zambetta, L. Cavedon, Video game personalisation techniques: A comprehensive survey, Entertainment Computing 5 (4) (2014) 211-218.

[6] M. Myślak, D. Deja, Developing game-structure sensitive matchmaking system for massive-multiplayer online games, in: M. L. Aiello, D. McFarland (Eds.), Social Informatics: SocInfo 2014 International Workshops, Barcelona, Spain, November 11, 2014, Revised Selected Papers, Springer International Publishing, Cham, 2015, pp. 200-208.

[7] M. Buchanan-Oliver, Y. Seo, Play as co-created narrative in computer game consumption: The hero's journey in warcraft iii, Journal of Consumer Behaviour 11 (6) (2012) 423-431. doi:10.1002/cb.392.

URL http://dx.doi.org/10.1002/cb.392

[8] Y. Francillette, L. Abrouk, A. Gouaich, A players clustering method to enhance the players' experience in multi-player games, in: Computer Games: AI, Animation, Mobile, Interactive Multimedia, Educational \& Serious Games (CGAMES), 18th International Conference on, IEEE, 2013, pp. 229-234.

[9] O. Delalleau, E. Contal, E. Thibodeau-Laufer, R. C. Ferrari, Y. Bengio, F. Zhang, Beyond skill rating: Advanced matchmaking in ghost recon 
online, Computational Intelligence and AI in Games, IEEE Transactions on 4 (3) (2012) 167-177.

[10] Y. Guo, S. Shen, O. Visser, A. Iosup, An analysis of online match-based games, in: Haptic Audio Visual Environments and Games (HAVE), IEEE International Workshop on, IEEE, 2012, pp. 134-139.

[11] M. Véron, O. Marin, S. Monnet, Matchmaking in multi-player on-line games: Studying user traces to improve the user experience, in: Proceedings of Network and Operating System Support on Digital Audio and Video Workshop, NOSSDAV '14, ACM, New York, NY, USA, 2014, pp. 7:7-7:12. doi:10.1145/2578260.2578265.

URL http://doi.acm.org/10.1145/2578260.2578265

[12] A. E. Elo, The rating of chessplayers, past and present, Arco Pub., 1978.

[13] R. Herbrich, T. Minka, T. Graepel, Trueskill?: A bayesian skill rating system, in: Advances in neural information processing systems, 2006, pp. 569-576.

[14] T. Graepel, R. Herbrich, Ranking and matchmaking, Game Developer Magazine 25 (2006) 34.

[15] T. Fritsch, B. Voigt, J. Schiller, The next generation of competitive online game organization, NetGames.

[16] R. A. Bradley, M. E. Terry, Rank analysis of incomplete block designs: I. the method of paired comparisons, Biometrika 39 (3/4) (1952) 324-345.

[17] T.-K. Huang, C.-J. Lin, R. C. Weng, A generalized bradley-terry model: From group competition to individual skill., in: Advances in Neural Information Processing Systems, 2004, pp. 601-608.

[18] J. E. Menke, T. R. Martinez, A bradley-terry artificial neural network model for individual ratings in group competitions, Neural computing and Applications 17 (2) (2008) 175-186.

[19] Z. Chen, Y. Sun, M. S. El-Nasr, T. D. Nguyen, Player skill decomposition in multiplayer online battle arenas, in: Meaningful Play, 2016.

[20] B. Bostan, A motivational framework for analyzing player and virtual agent behavior, Entertainment Computing 1 (3) (2010) 139-146. 
[21] Z. Chen, M. S. El-Nasr, A. Canossa, J. Badler, S. Tignor, R. Colvin, Modeling individual differences through frequent pattern mining on roleplaying game actions, in: Eleventh Artificial Intelligence and Interactive Digital Entertainment Conference, 2015.

[22] A. Nagle, P. Wolf, R. Riener, Towards a system of customized video game mechanics based on player personality: Relating the big five personality traits with difficulty adaptation in a first-person shooter game, Entertainment Computing 13 (2016) 10-24.

[23] C. Eggert, M. Herrlich, J. Smeddinck, R. Malaka, Classification of player roles in the team-based multi-player game dota 2, in: International Conference on Entertainment Computing, Springer, 2015, pp. 112-125.

[24] G. Jiménez-Dıaz, H. D. Menéndez, D. Camacho, P. A. González-Calero, Predicting performance in team games, II for Systems, C. Technologies of Information, and Communication, ICAART (2011) 401-406.

[25] K. B. Shores, Y. He, K. L. Swanenburg, R. Kraut, J. Riedl, The identification of deviance and its impact on retention in a multiplayer game, in: Proceedings of the 17th ACM conference on Computer supported cooperative work \& social computing, ACM, 2014, pp. 1356-1365.

[26] J. Riegelsberger, S. Counts, S. D. Farnham, B. C. Philips, Personality matters: Incorporating detailed user attributes and preferences into the matchmaking process, in: System Sciences, 2007. HICSS 2007. 40th Annual Hawaii International Conference on, IEEE, 2007, pp. 87-87. doi:10.1109/HICSS.2007.434.

[27] S. Prugsamatz, B. Lowe, F. Alpert, Modelling consumer entertainment software choice: An exploratory examination of key attributes, and differences by gamer segment, Journal of Consumer Behaviour 9 (5) (2010) 381-392.

[28] A. E. Roth, M. A. Sotomayor, Two-Sided Matching: A Study in GameTheoretic Modeling and Analysis, Econometric Society Monographs, Cambridge University Press, 1990.

[29] G. Shmueli, O. R. Koppius, Predictive analytics in information systems research, MIS Quarterly 35 (3) (2011) 553-572. 
[30] F. Collopy, M. Adya, J. S. Armstrong, Principles for examining predictive-validity - the case of information systems spending forecasts, Information Systems Research 5 (2) (1994) 170-179.

[31] V. Sethi, R. C. King, Nonlinear and noncompensatory models in user information satisfaction measurement, Information Systems Research 10 (1) (1999) 87-96.

[32] J.-H. Wu, S.-C. Wang, H.-H. Tsai, Falling in love with online games: The uses and gratifications perspective, Computers in Human Behavior 26 (6) (2010) 1862-1871.

[33] R. Van De Bovenkamp, S. Shen, A. Iosup, F. Kuipers, Understanding and recommending play relationships in online social gaming, in: Communication Systems and Networks (COMSNETS), Fifth International Conference on, IEEE, 2013, pp. 1-10.

[34] J. L. Sherry, K. Lucas, B. S. Greenberg, K. Lachlan, Video game uses and gratifications as predictors of use and game preference, Playing video games: Motives, responses, and consequences 24 (2006) 213-224. 


\section{Appendix A. Tables}

Table A.1: Descriptive Statistics: Round-Level Data

\begin{tabular}{|c|c|c|c|c|c|c|}
\hline Variable & Description & Obs & Mean & Std. Dev. & Min & Max \\
\hline $\begin{array}{l}\text { Mean Proportion of Round } \\
\text { Completed } \\
\text { Gaming Skills }\end{array}$ & $\begin{array}{l}\text { Average proportion of round completed by } \\
\text { players. }\end{array}$ & 30156207 & .579 & .155 & .001 & .994 \\
\hline Mean Rank Start & $\begin{array}{l}\text { Average rank of players at the start of the } \\
\text { game round. }\end{array}$ & 30156207 & 44.943 & 18.911 & 0 & 145 \\
\hline Std Dev Rank Start & $\begin{array}{l}\text { Standard deviation of player ranks at the } \\
\text { start of the game round. }\end{array}$ & 30156196 & 23.366 & 10.495 & 0 & 87.681 \\
\hline Mean Kills per Second & $\begin{array}{l}\text { Average number of kills made by a player per } \\
\text { second during the round. }\end{array}$ & 30156207 & .008 & .005 & 0 & .142 \\
\hline Std Dev Kills per Second & $\begin{array}{l}\text { Standard deviation of the number of kills } \\
\text { made by a player per second during the } \\
\text { round. }\end{array}$ & 30156196 & .006 & .003 & 0 & .118 \\
\hline Mean Non Combat Score & $\begin{array}{l}\text { Average score earned by players in a given } \\
\text { round derived from non-combat (e.g. healing } \\
\text { or repairing). }\end{array}$ & 30156207 & 2215.959 & 2062.832 & 0 & 159178.6 \\
\hline Std Dev Non Combat Score & $\begin{array}{l}\text { Standard deviation of players' non-combat } \\
\text { scores in round. }\end{array}$ & 30156196 & 3262.605 & 2923.806 & 0 & 153665 \\
\hline Max Simultaneous Players & $\begin{array}{l}\text { Maximum number of players active in the } \\
\text { game round at any single point in time. }\end{array}$ & 30156207 & 24.919 & 12.711 & 2 & 64 \\
\hline Proportion Role (Assault) & $\begin{array}{l}\text { Proportion of players who adopt the 'Assault' } \\
\text { role at some point during the round. }\end{array}$ & 30156207 & .396 & .211 & 0 & 1 \\
\hline Proportion Role (Recon) & $\begin{array}{l}\text { Proportion of players who adopt the 'Recon' } \\
\text { role at some point during the round. }\end{array}$ & 30156207 & .317 & .174 & 0 & 1 \\
\hline Proportion Role (Engineer) & $\begin{array}{l}\text { Proportion of players who adopt the 'Engi- } \\
\text { neer' role at some point during the round. }\end{array}$ & 30156207 & .45 & .227 & 0 & 1 \\
\hline Proportion Role (Support) & $\begin{array}{l}\text { Proportion of players who adopt the 'Sup- } \\
\text { port' role at some point during the round. }\end{array}$ & 30156207 & .301 & .158 & 0 & 1 \\
\hline $\begin{array}{l}\text { Proportion Vehicle (Armored } \\
\text { Land) }\end{array}$ & $\begin{array}{l}\text { Proportion of players who make use of an ar- } \\
\text { mored land vehicle at some point during the } \\
\text { round. }\end{array}$ & 30156207 & .277 & .265 & 0 & 1 \\
\hline $\begin{array}{l}\text { Proportion Vehicle (Unarmored } \\
\text { Land) }\end{array}$ & $\begin{array}{l}\text { Proportion of players who make use of an un- } \\
\text { armored land vehicle at some point during } \\
\text { the round. }\end{array}$ & 30156207 & .155 & .195 & 0 & 1 \\
\hline
\end{tabular}


Table A.1: Descriptive Statistics: Round-Level Data

\begin{tabular}{|c|c|c|c|c|c|c|}
\hline Variable & Description & Obs & Mean & Std. Dev. & Min & Max \\
\hline Proportion Vehicle (Jet) & $\begin{array}{l}\text { Proportion of players who make use of a jet } \\
\text { at some point during the round. }\end{array}$ & 30156207 & .066 & .132 & 0 & 1 \\
\hline Proportion Vehicle (Helicopter) & $\begin{array}{l}\text { Proportion of players who make use of a he- } \\
\text { licopter at some point during the round. }\end{array}$ & 30156207 & .155 & .222 & 0 & 1 \\
\hline Proportion Vehicle (Boat) & $\begin{array}{l}\text { Proportion of players who make use of a boat } \\
\text { at some point during the round. }\end{array}$ & 30156207 & .01 & .039 & 0 & 1 \\
\hline Game Mode (Conquest) & $\begin{array}{l}\text { Round is played using a Conquest game } \\
\text { mode. }\end{array}$ & 30156207 & .247 & .431 & 0 & 1 \\
\hline Game Mode (Rush) & Round is played using a Rush game mode. & 30156207 & .032 & .177 & 0 & 1 \\
\hline $\begin{array}{l}\text { Game Mode (Team Death } \\
\text { Match) }\end{array}$ & $\begin{array}{l}\text { Round is played using a Team Death Match } \\
\text { game mode. }\end{array}$ & 30156207 & .015 & .122 & 0 & 1 \\
\hline Level (MP1) & Round is played on the MP001 map. & 30156207 & .08 & 272 & 0 & 1 \\
\hline Level (MP3) & Round is played on the MP003 map. & 30156207 & .039 & .195 & 0 & 1 \\
\hline Level (MP11) & Round is played on the MP011 map. & 30156207 & .069 & .254 & 0 & 1 \\
\hline Level (MP12) & Round is played on the MP012 map. & 30156207 & .079 & .27 & 0 & 1 \\
\hline Level (MP13) & Round is played on the MP013 map. & 30156207 & .056 & .23 & 0 & 1 \\
\hline Level (MP17) & Round is played on the MP017 map. & 30156207 & .104 & .305 & 0 & 1 \\
\hline $\begin{array}{l}\text { Level (MP Sub) } \\
\text { Complementary Attributes }\end{array}$ & Round is played on the MPSubway map. & 30156207 & .149 & .356 & 0 & 1 \\
\hline Mean Age & $\begin{array}{l}\text { Average age of a player participating in the } \\
\text { round. }\end{array}$ & 30156207 & 29.946 & 2.105 & 17 & 80 \\
\hline Std Dev Age & $\begin{array}{l}\text { Standard deviation of player ages within the } \\
\text { round. }\end{array}$ & 30156196 & 9.56 & 2.125 & 0 & 41.012 \\
\hline Number of Countries & $\begin{array}{l}\text { Number of individual countries in which play- } \\
\text { ers participating in the round are based. }\end{array}$ & 30156207 & 10.989 & 6.588 & 1 & 57 \\
\hline $\begin{array}{l}\text { Proportion of Male Players } \\
\text { Round Characteristics }\end{array}$ & Proportion of male players within the round. & 30156207 & .099 & .197 & 0 & 9.5 \\
\hline Platform (PC) & $\begin{array}{l}\text { Proportion of rounds in which } \mathrm{PC} \text { is used as } \\
\text { gaming platform. }\end{array}$ & 30156207 & .361 & .48 & 0 & 1 \\
\hline Platform (PS3) & $\begin{array}{l}\text { Proportion of rounds in which PS3 is used as } \\
\text { gaming platform. }\end{array}$ & 30156207 & .318 & .466 & 0 & 1 \\
\hline Platform (XBOX) & $\begin{array}{l}\text { Proportion of rounds in which Xbox360 is } \\
\text { used as gaming platform. }\end{array}$ & 30156207 & .321 & .467 & 0 & 1 \\
\hline
\end{tabular}


Table A.2: Regression Results for Round-Level Data

\begin{tabular}{|c|c|c|c|}
\hline VARIABLES & $\begin{array}{c}(\mathrm{I}) \\
\text { LINEAR } \\
\text { Coefficient } \\
\end{array}$ & \multicolumn{2}{|c|}{$\begin{array}{c}(\text { II }) \\
\text { NON-LINEAR } \\
+\end{array}$} \\
\hline & \multicolumn{3}{|c|}{ Proportion of Round Completed } \\
\hline $\begin{array}{l}\text { Gaming Skills } \\
\text { Mean Rank Start }\end{array}$ & $-0.001^{*}$ & $-0.001 * * *$ & $0.000 * * *$ \\
\hline Std Dev Rank Start & $\begin{array}{l}(0.000) \\
0.001 * * * \\
(0.000)\end{array}$ & $\begin{array}{l}(0.000) \\
0.001 * * * \\
(0.000)\end{array}$ & $\begin{array}{l}(0.000) \\
-0.000 * * * \\
(0.000)\end{array}$ \\
\hline Mean Kills per Second & $\begin{array}{l}27.969^{* * *} \\
(0.019)\end{array}$ & $\begin{array}{l}36.899^{* * * *} \\
(0.178)\end{array}$ & $\begin{array}{l}-777.908^{* * *} \\
(18.252)\end{array}$ \\
\hline Std Dev Kills per Second & $\begin{array}{l}-1.188^{* * *} \\
(0.040)\end{array}$ & $\begin{array}{l}-4.738^{* * *} \\
(0.094)\end{array}$ & $\begin{array}{l}354.664^{* * *} \\
(27.564)\end{array}$ \\
\hline Mean Non Combat Score & $\begin{array}{l}0.000^{* * *} \\
(0.000)\end{array}$ & $\begin{array}{l}0.000^{* * *} \\
(0.000)\end{array}$ & $\begin{array}{l}-0.000^{* * *} \\
(0.000)\end{array}$ \\
\hline Std Dev Non Combat Score & $\begin{array}{l}-0.000^{* * *} \\
(0.000)\end{array}$ & $\begin{array}{l}-0.000^{* * *} \\
(0.000)\end{array}$ & $\begin{array}{l}0.000^{* * *} \\
(0.000)\end{array}$ \\
\hline $\begin{array}{l}\text { Inherent Attributes } \\
\text { Max Simultaneous Players }\end{array}$ & $\begin{array}{l}0.001 * * * \\
(0.000)\end{array}$ & $\begin{array}{l}0.001 * * * \\
(0.000)\end{array}$ & $\begin{array}{l}-0.000^{* * *} \\
(0.000)\end{array}$ \\
\hline Proportion Role (Assault) & $\begin{array}{l}0.043^{* * *} \\
(0.000)\end{array}$ & $\begin{array}{l}0.023^{* * *} \\
(0.000)\end{array}$ & $\begin{array}{l}-0.060^{* * *} \\
(0.001)\end{array}$ \\
\hline Proportion Role (Recon) & $\begin{array}{l}0.095^{* * *} \\
(0.000)\end{array}$ & $\begin{array}{l}0.083^{* * *} \\
(0.000)\end{array}$ & $\begin{array}{l}-0.019^{* * *} \\
(0.001)\end{array}$ \\
\hline Proportion Role (Engineer) & $\begin{array}{l}0.071^{* * *} \\
(0.000)\end{array}$ & $\begin{array}{l}0.055^{* * *} \\
(0.000)\end{array}$ & $\begin{array}{l}-0.025^{* * *} \\
(0.001)\end{array}$ \\
\hline Proportion Vehicle (Armored Land) & $\begin{array}{l}0.137^{* * *} \\
(0.000)\end{array}$ & $\begin{array}{l}0.167^{* * *} \\
(0.000)\end{array}$ & $\begin{array}{l}-0.138^{* * *} \\
(0.001)\end{array}$ \\
\hline Proportion Vehicle (Unarmored Land) & $\begin{array}{l}0.133^{* * * *} \\
(0.000)\end{array}$ & $\begin{array}{l}0.154^{* * *} \\
(0.000)\end{array}$ & $\begin{array}{l}-0.139^{* * *} \\
(0.001)\end{array}$ \\
\hline Proportion Vehicle (Jet) & $\begin{array}{l}0.050^{* * * *} \\
(0.000)\end{array}$ & $\begin{array}{l}0.034^{* * *} \\
(0.001)\end{array}$ & $\begin{array}{l}0.070^{* * *} \\
(0.002)\end{array}$ \\
\hline Proportion Vehicle (Helicopter) & $\begin{array}{l}0.087^{* * *} \\
(0.000)\end{array}$ & $\begin{array}{l}0.209^{* * *} \\
(0.000)\end{array}$ & $\begin{array}{l}-0.265^{* * *} \\
(0.001)\end{array}$ \\
\hline Proportion Vehicle (Boat) & $\begin{array}{l}0.142^{* * *} \\
(0.001)\end{array}$ & $\begin{array}{l}0.141^{* * *} \\
(0.002)\end{array}$ & $\begin{array}{l}-0.125^{* * *} \\
(0.005)\end{array}$ \\
\hline Game Mode (Conquest) & $\begin{array}{l}-0.003^{* * *} \\
(0.000)\end{array}$ & $\begin{array}{l}-0.005^{* * *} \\
(0.000)\end{array}$ & \\
\hline Game Mode (Rush) & $\begin{array}{l}0.042^{* * *} \\
(0.000)\end{array}$ & $\begin{array}{l}0.040^{* * *} \\
(0.000)\end{array}$ & \\
\hline Game Mode (Team Death Match) & $\begin{array}{l}-0.011 * * * \\
(0.000)\end{array}$ & $\begin{array}{l}-0.004^{* * *} \\
(0.000)\end{array}$ & \\
\hline
\end{tabular}


Table A.2: Regression Results for Round-Level Data

\begin{tabular}{|c|c|c|c|}
\hline VARIABLES & $\begin{array}{c}(\mathrm{I}) \\
\text { LINEAR } \\
\text { Coefficient }\end{array}$ & \multicolumn{2}{|c|}{$\begin{array}{c}(\text { III }) \\
\text { NON-LINEAR }\end{array}$} \\
\hline Level (MP1) & $0.036 * * *$ & $0.027^{* * *}$ & \\
\hline & $(0.000)$ & $(0.000)$ & \\
\hline Level (MP3) & $0.034^{* * *}$ & $0.033^{* * *}$ & \\
\hline Level (MP11) & $\begin{array}{c}(0.000) \\
0.053^{* * *}\end{array}$ & $\begin{array}{l}(0.000) \\
0.044^{* * *}\end{array}$ & \\
\hline Level (MP12) & $(0.000)$ (0.015*** & $(0.000)$ & \\
\hline Level (MP13) & $\begin{array}{l}(0.000) \\
0.035^{* * *} \\
(0.000)\end{array}$ & $\begin{array}{l}(0.000) \\
0.012^{* * *} \\
(0.000)\end{array}$ & \\
\hline Level (MP17) & $-0.049^{* * *}$ & $-0.050^{* * *}$ & \\
\hline Level (MP Sub) & $\begin{array}{l}0.045^{* * * *} \\
(0.000)\end{array}$ & $\begin{array}{l}0.054^{* * *} \\
(0.000)\end{array}$ & \\
\hline $\begin{array}{l}\text { Complementary Attributes } \\
\text { Mean Age }\end{array}$ & $\begin{array}{l}0.005^{* * *} \\
(0.000)\end{array}$ & $\begin{array}{l}0.005^{* * *} \\
(0.000)\end{array}$ & $\begin{array}{l}0.000^{* * *} \\
(0.000)\end{array}$ \\
\hline Std Dev Age & $\begin{array}{l}-0.004^{* * *} \\
(0.000)\end{array}$ & $\begin{array}{l}-0.005^{* * *} \\
(0.000)\end{array}$ & $\begin{array}{l}0.001^{* * *} \\
(0.000)\end{array}$ \\
\hline Number of Countries & $\begin{array}{l}-0.005^{* * *} \\
(0.000)\end{array}$ & $\begin{array}{l}-0.005^{* * *} \\
(0.000)\end{array}$ & $\begin{array}{l}0.000 * * * \\
(0.000)\end{array}$ \\
\hline Proportion of Male Players & $\begin{array}{l}-0.069^{* * *} \\
(0.000)\end{array}$ & $\begin{array}{l}-0.041^{* * *} \\
(0.000)\end{array}$ & \\
\hline $\begin{array}{l}\text { Observations } \\
\text { R-squared }\end{array}$ & $\begin{array}{r}30,156,196 \\
0.584\end{array}$ & $\begin{array}{r}30,156,196 \\
0.657\end{array}$ & \\
\hline $\begin{array}{l}\text { Robust standard errors in } p \\
\text { The following represent adc } \\
\text { tioned specifications: Dumr } \\
\text { round was completed), whic } \\
\text { in the round at any given ti } \\
\text { in the round opened an acce } \\
\text { specific game, how much ( } \\
\text { and the standard deviation } \\
\text { the start date of a round. }\end{array}$ & $\begin{array}{l}{ }^{* *} p<0.01, * * \\
\text { ol variables use } \\
\mathrm{s} \text { whether or no } \\
\text { as used, the ma } \\
\text { and std dev of } \\
\text { company and } \\
\text { tomers have spe } \\
\text { ditures as of th }\end{array}$ & $\begin{array}{l}<0.05{ }^{*} p< \\
\text { in each of the } \\
\text { the round wa: } \\
\text { imum amount } \\
\text { the date on wh } \\
n \text { which they st } \\
\text { it with the con } \\
\text { time the data }\end{array}$ & $\begin{array}{l}1 \\
\text { previously men- } \\
\text { completed }(1= \\
\text { players allowed } \\
\text { h the customers } \\
\text { rted to play the } \\
\text { pany on average } \\
\text { was pulled, and }\end{array}$ \\
\hline
\end{tabular}


Table A.3: Comparison between pure skills-based matching and nested matching

\begin{tabular}{|c|c|c|}
\hline \multirow[b]{3}{*}{ Fit Validity } & Skill-based Matching & Nested Matching \\
\hline & \multicolumn{2}{|l|}{$R^{2}$} \\
\hline & 7.4 & 20.91 \\
\hline \multicolumn{3}{|l|}{ Predictive Validity } \\
\hline Est 1 & 31.19 & 20.71 \\
\hline Est 2 & 12.31 & 20.85 \\
\hline Est 3 & 31.04 & 20.66 \\
\hline Est 4 & 11.26 & 21.17 \\
\hline Est 5 & 2.27 & 21.18 \\
\hline Mean & 17.614 & 20.914 \\
\hline
\end{tabular}

\section{Appendix B. Figures}

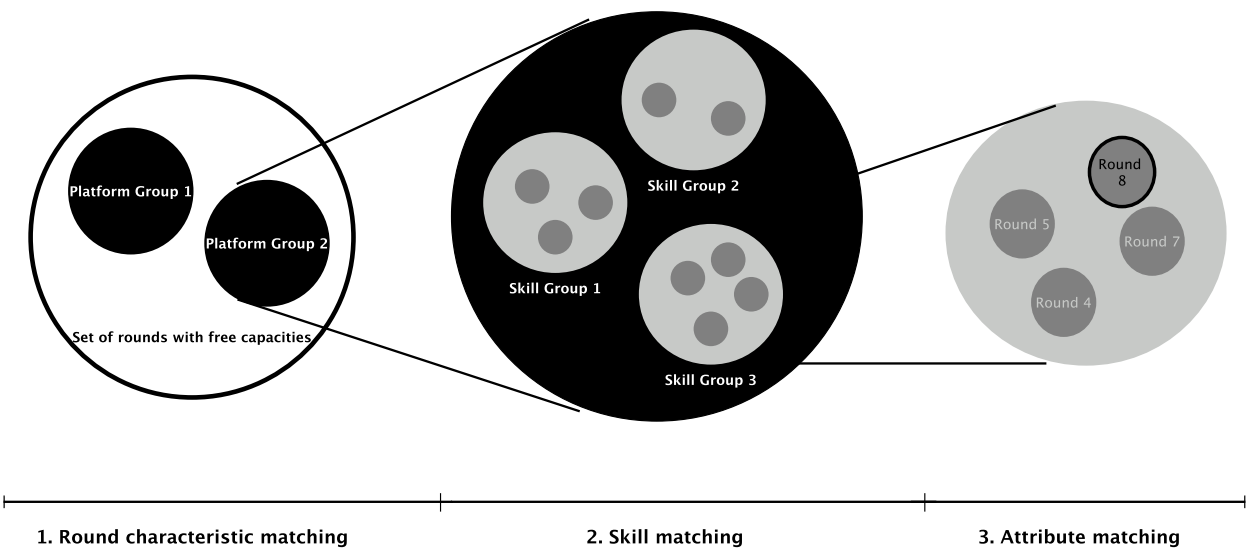

Figure B.1: Illustration of nested matching 